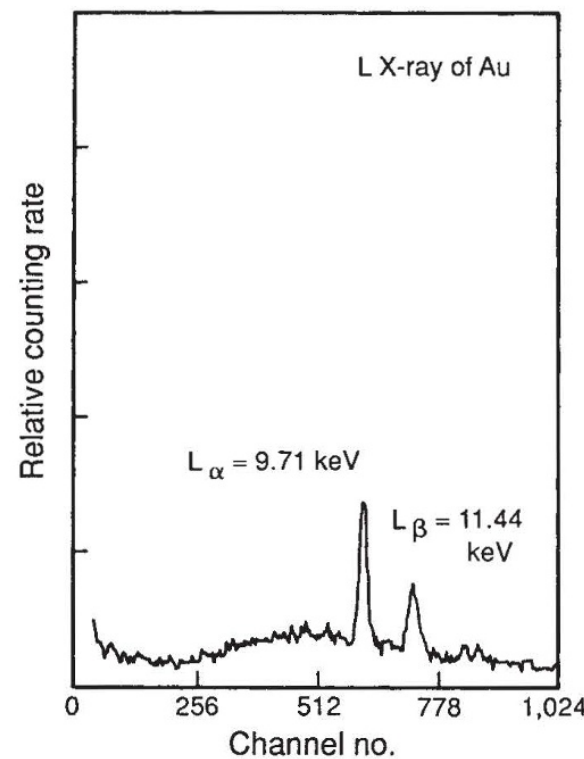

Spectrum of $\mathrm{Au} L \mathrm{X}$-ray detected with a $\mathrm{Si}(\mathrm{Li})$ X-ray spectrometer.

not produced, thus providing an upper limit for the energy of the ejected electrons.

The X-ray generator is a small vacuum cryostat with a liquid nitrogen reservoir, a thin window and a heater/cold finger. One end of the heater/cold finger is connected to a liquid nitrogen reservoir and the other faces the window. An ambient vacuum of $5 \times 10^{-5}$ torr is sufficient. The crystal is placed on the end of the heater/cold finger with the positive end approximately perpendicular to the target and parallel to the window of the cryostat. A simple spring clip holds the crystal in place. The heater is a $47-\Omega$ wire-wound resistor inside a brass tube that is the cold finger. The temperature of the cold finger is monitored with a thermocouple and the cryostat window is $4.5 \mathrm{mg} \mathrm{cm} \mathrm{m}^{-2}$ aluminium.

A typical $\mathrm{CsNO}_{3}$ crystal grown in an aqueous solution is $0.2 \times 0.3 \times 0.8 \mathrm{~cm}$ and is very fragile. When these crystals are cooled for the first time, they fracture and fragment. To prevent separation after fracturing, crystals are covered with a thin layer of epoxy. The ends may or may not be covered, as a thin layer of epoxy on the ends has little effect on the production of X-rays. The electrons accelerated by the crystal come from the ionization of molecules of the residual gases in the system.

The polarity of each crystal is determined by suspending it in liquid nitrogen in an electric field of known polarity and observing the alignment of the crystal with the electric field as it cools. If any part of the crystal is not covered with epoxy, all water must be removed immediately following this procedure.

To produce $\mathrm{X}$-rays the heater is turned on and off, cycling the crystal between approximately 77 and $273 \mathrm{~K}$.
The cycle time is about $5 \mathrm{~min}$, with about 1 min of X-ray production. With a thin window $\mathrm{NaI}(\mathrm{Tl})$ detector and a gold foil $0.5 \mathrm{~cm}$ from the crystal, 150,000 photons are detected in less than $1 \mathrm{~min}$. No apparent reduction of X-rays is observed after repeated temperature cycles or if the crystal is maintained for long periods at either 77 or $300 \mathrm{~K}$.

James D. Brownridge

Department of Physics, Applied Physics, and Astronomy,

State University of New York,

PO Box 6000 , Binghamton,

New York 13902, USA

1. Rosenblum, B., Braunlich, P. \& Carrico, J. P. Appl. Phys. Lett. 25, 1 (1974).

2. Kalent'ev, V. A., Kortov, S. V. \& Zatespin, A. F. Sov. Tech. Phys. Lett. 13(11) (1987)

3. Rosenman, G. 1., Rez, I. S., Chepelev, Yu. L. \& Angert N. B. Sov. Phys. Tech. Phys. 26(2) (1981)

4. Kortov, V. S. et al. Sov. Phys. Tech. Phys. 25(9) (1980).

5. Minakova, E. V., Tirkhomirova, N. A. \& Khrustalev, Yu. A. Phys. Chem. Mech. Surf. 5(7) 1861-1864 (1990)

6. Rosenman, G. I., Pechorskii, V. I., Chepelev, Yu. L., Boikova, E. I. \& Issakova, L. E. Phys. Stat. Sol. 120, 667 (1983).

7. Biedrzycki, K. Ferroelectrics 119, 33 (1991)

8. Brownridge, J. D., Telesca, A. J., Stannard, C. R. \& O'Brien, T. P. Phys. Tech. 28(7), 482 (1990)

\section{APC mutations}

SIR - The function of multimeric proteins, or multiprotein aggregates, can be altered by inhibitory polypeptides produced through the action of dominant negative mutations ${ }^{1}$. The discovery of loci involved in colon cancer ${ }^{2}$ which are dominant for polyp formation prompted Bourne to propose that these mutations exert their phenotype by this mechanism $^{3}$. However, in a more recent paper, Groden et al. ${ }^{4}$ argued that the nature of the observed mutational events in these patients with adenomatous polyposis coli (APC), which were found to be nonsense mutations and frameshifts, is inconsistent with such a model (the observed mutational events are incompatible with a dominant negative phenotype).

The consequence of such mutations, however, need not only be the inactivation of the gene and its protein product; through the process of translational reinitiation they can also alter the function of the product. In bacteria ${ }^{5}$, nonsense and frameshift events that create

\section{Scientific Correspondence}

Scientific Correspondence is a relatively informal section of Nature in which matters of general scientific interest, not necessarily those arising from papers appearing in Nature, are published. Because there is space to print only a small proportion of the letters received, priority is usually given according to general interest and topicality, to contributions of fewer than 500 words, and to contributions using simple language. or activate barriers to translation result in the production of dominant restart polypeptides in genes or proteins that exhibit an appropriate structure and function. This process provides a ready explanation for the APC results, namely, the carboxy-terminal fragments so generated by reinitiated translation interfere with the normal functioning of APC and/or MCC (mutated in colon cancer) protein complexes. Indeed, such a phenomenon may be particularly important in the alteration of the function of proteins insensitive to amino-acid substitution. Such functions would be sensitive to various mutational events that alter the reading frame (single-base frameshifts, deletions, duplications and rearrangements) while providing only a limited target for missense mutations. These mutational events have been neglected of late because of the predominance of single-base substitutions in the oncogene/suppressor gene systems so far characterized.

\section{Alasdair Gordon}

Section de Biologie,

Institut Curie,

26 rue d'Ulm,

\section{Paris Cédex, France}

1. Herskowitz, I. Nature 329, 219-222 (1987).

2. Kinzler, K. W. et al. Science 251, 1366-1370 (1991)

3. Bourne, H. R. Nature 351, 188-190 (1991).

4. Groden, J. et al. Cell 66, 589-600 (1991)

5. Sarabhai, A. \& Brenner, S. J. molec. Biol. 27, 145-162 (1967).

\section{Time machine from dust}

SIR - Allen and Simon ${ }^{1}$ explain how a pair of cosmic strings, if they exist, could form a time machine. However, there are several other space-time metrics of general relativity which have this property, some of them quite simple.

Probably the simplest is the one discovered long ago by van Stockum ${ }^{2,3}$. This refers to the gravitational field outside an infinitely long circular cylinder in steady, rigid rotation. The cylinder is made of dust, that is, matter whose constituent parts have no interaction except their mutual gravitation. The properties of the system are governed by the mass per unit length, $m$. When $m$ is not very large the gravitational field outside the matter is just that of a static infinite cylinder. This is surprising because it means that, contrary to expectations from Mach's principle, the rotating matter does not drag the exterior spacetime with it.

When $m$ is large (in fact, greater than $2 \times 10^{27} \mathrm{~g} \mathrm{~cm}^{-1}$ ), the rotating matter does drag the exterior space-time, and closed time-like lines exist outside the cylinder. Therefore an observer, using rockets, could travel into his past: the 